\section{SCREENING AND MANAGEMENT OF NEONATAL JAUNDICE BY TRANSCUTANEOUS BILIRUBIN}

\section{E. Tiberi, L. Giordano, C. Romagnoli, E. Zecca}

Department of Pediatrics, Division of Neonatology, Policlinico Gemelli UCSC, Rome, Italy

Objective: The aim of this study was to assess the validity of our previously developed skin bilirubin nomogram in predicting severe hyperbilirubinemia (> $17 \mathrm{mg} / \mathrm{dl}$ ) in healthy term and late preterm newborns.

Methods: This multicenter study was conducted from February 2009 to December 2009 in the well-baby nurseries of five centres of Rome. The predictive ability of the nomogram for transcutaneous bilirubin (TCB) values in the first 96 hours of life was prospectively assessed in 2167 neonates with gestational age $\geq 35$ weeks. Skin bilirubin was measured with the Bilichek and serum bilirubin was contemporary assayed by a spectrophotometric method. 1074 neonates had more than one measurements giving a total of 3241 values.

Results: The mean TSB values was $8,3 \pm 3 \mathrm{mg} / \mathrm{dl}$, while the mean TCB values was $9,5 \pm 3,3 \mathrm{mg} / \mathrm{dl}$. A sensitivity of $96,4 \%$ and a negative predictive value (NPV) of $99.9 \%$ were obtained with a single bilirubin determination applying the $75^{\circ}$ percentile of our nomogram. Two consecutive TCB determinations identified all infants reaching TSB values $>17 \mathrm{mg} /$ dl (100 $\%$ of sensitivity and $100 \%$ of NPV).

Conclusion: The $75^{\circ}$ percentile of the skin bilirubin nomogram for the European population in the first 96 hours of life is able to predict all neonates at risk of severe hyperbilirubinemia. It is a reliable guide for intervention and follow-up, and allows a safe discharge from the hospital, also reducing a large number of blood samples.

\section{6}

\section{NECROTIZING ENTEROCOLITIS IN PRETERM INFANTS ON DIFFERENT FEEDING PROTOCOL}

\author{
V. Marinkovic, N. Bozinovic-Prekajski, \\ V. Hajdarpasic
}

Institute for Neonatology, Belgrade, Serbia

Background and aims: Feeding with human milk $(H M)$ is in correlation with many useful effects and represents key component in strategy of enteral feeding of preterm infants. Objective of this research was to determine incidence of necrotizing enterocolitis (NEC) in structure of morbidity and relative risk (RR) of acquiring NEC in preterm infants fed with fortified human milk (FHM) and preterm formula (PF) respectively.

Methods: Total of 100 preterm infants of gestational age less than 34 weeks were included in this prospective analysis. Subjects were divided in two groups: group I $(n=50)$ fed with FHM (FM 85 Nestle) and group II $(n=50)$ fed with PF (preNAN Nestle) in case where lactation wasn't established, for 4 weeks. NEC was diagnosed if presence of intestinal pneumatosis on X-ray was verified (Bell's stage II and III).

Results: Initially, examined groups did not differ considering the gestational age, Apgar score and antropometric parameters at birth. Subjects fed with FHM reached full enteral feeding earlier compared to PF fed group $(p<0,05)$. Incidence of NEC in FHM fed group was $2 \%$, and in PF fed group 6\% $(p>0,05)$. In FHM fed group RR=0,33, $95 \% \mathrm{Cl}(0,36-3,097)$, which means that risk of acquiring NEC is $33 \%$ less compared to risk in $\mathrm{PF}$ fed group.

Conclusion: There is no statistically significant difference between these two groups in incidence of NEC, but RR indicates a smaller risk of NEC in FHM fed group

\section{7}

\section{PARATHYROID HORMONE RELATED PROTEIN (PTHRP) IN PRETERM HUMAN MILK}

A. Ben Tov' ${ }^{1,2}$, R. Lubetzky ${ }^{1,2}$, S. Ben Or ${ }^{1}$, T. Taxir', G. Zeidenberg ${ }^{1}$, S. Dollberg, ${ }^{2,3}$, D. Mandel ${ }^{2,3}$, I. Berger ${ }^{2,3}$

${ }^{1}$ Pediatric, Sourasky Medical Center, ${ }^{2}$ Sackler

Faculty of Medicine, Tel-Aviv University, ${ }^{3}$ Neonatology, Sourasky Medical Center, Tel Aviv, Israel

Background: Parathyroid hormone-related protein (PTHrP) has the ability to activate parathyroid hormone receptors and cause hypercalcaemia. In a previous study we demonstrated high levels of PTHrP in both term and preterm human milk (HM) (Breastfeeding Medicine, 2009). PTHrP intestinal absorption and its influence upon calcium homeostasis of the preterm infant (PTI) has not been studied yet. 
Objective: To assess the correlation between PTHrP level in preterm HM and PTHrP in maternal and neonatal serum.

Design/methods: We collected samples of expressed $\mathrm{HM}$ obtained from 16 mothers of preterm infants (25-34 weeks' gestation). In addition, blood samples from both mothers and infants were drawn. All samples were collected at days 2 and 10 postpartum. PTHrP levels were measured by twosite immunoradiometric assay. Blood calcium (Ca), Phosphorus $(P)$ and alkaline phosphatase (ALP) levels were also measured.

Results: Both maternal and neonatal PTHrP serum levles did not vary after 10 days of breastfeeding. No correlation was found between PTHrP level in HM and serum PTHrP, Ca, P and ALP of the breastfed PTI.

Conclusions: Despite high levels of PTHrP in preterm HM, serum levels of PTHrP of the breast fed PTI do not increase over time. We found no correlation between PTHrP in $\mathrm{HM}$ and neonatal Ca serum concentration. We speculate that PTHrP intestinal absorption in the PTI is not significant.

\section{8}

\section{GASTROESOPHAGEAL REFLUX IN CRITICALLY ILL CHILDREN}

M.J. Solana, C. Sanchez, J. Lopez-Herce, M. Crespo, A. Sanchez, J. Urbano, M. Botran, J.M. Bellon, A. Carrillo, Gregorio Marañon PICU

\section{Gregorio Marañón General University Hospital,} Madrid, Spain

Introduction: Gastroesophageal reflux (GER) is common in children, but its incidence in critically ill pediatric patients is unknown. Our objective was to study the incidence and type of GER in critically ill children.

Methods: Prospective observational study in critically ill children aged 1 month to 14 years. A multichannel catheter (MII) was inserted at the admittance in the PICU. This catheter has 6 channels for esophageal multichannel intraluminal impedance (MII) and 2 channels for esophageal and gastric $\mathrm{pH}$ measurement. Patients were studied during 48 hours.

Results: Twenty-four patients (mean age, 16.4 months) were studied. MII detected the presence of GER in $80 \%$ of patients, whereas $\mathrm{pH}$ monitoring only detected $20 \%$, although the correlation between the two was good. MII detected 320 episodes of GER (58\% acid, 42\% alkaline; 143 were gas type) whereas $\mathrm{pH}$ monitoring detected 26 episodes $(100 \%$ acid). Patients with most episodes of GER were children between 7 and 12 months. The median duration of the longest episodes of GER was 4.1 minutes by $\mathrm{pH}$ monitoring and 2.3 minutes by MII. There was no relationship between the PRISM or PIM scores and the number of episodes of GER. There were no differences in the number or types of reflux according to the administration of epinephrine, dopamine, midazolam, fentanyl, or vecuronium.

Conclusions: The incidence of GER in critically ill children is high. MII is more sensitive than $\mathrm{pH}$ monitoring because many GER episodes are alkaline or weak acid.

\section{9}

\section{POLYMORPHIC VARIANTS OF UGT1A1 IN NEONATAL JAUNDICE IN SOUTHERN BRAZIL}

C.G. Carvalho ${ }^{1}$, S.M. Castro ${ }^{2}$, A.P. Santin², L.A. de Azevedo², M.L. Pereira ${ }^{3}$, R. Giugliani ${ }^{3}$

${ }^{1}$ UFRGS - HCPA, ${ }^{2}$ UFRGS, ${ }^{3}$ Genetic Service, UFRGS - HCPA, Porto Alegre, Brazil

Background and aims: Neonatal jaundice seems a benign condition but babies must be monitored in order to identify those who are at higher risk of encephalopathy. Abnormalities in hepatic conjugation of bilirubin were proposed in those cases - polymorphisms of the hepatic enzyme UGT1A1. Herein, we estimated the frequency of alleles and of genotypes of the promoter region of UGT1A1 gene in newborns and evaluated its association with severe hyperbilirubinemia

Methods: Case-control study, including all neonates who were in phototherapy at NICU/HCPA from March to December 2007, born > 35w of gestational age and weight $>2000 \mathrm{~g}$. Controls were neonates not jaundiced. Informed consent form applied. PCR performed and analyzed in GeneMapper ${ }^{\circledR}$ program

Results (490 NBs included): There were 7 of the 10 possible UGT1A1 genotypes identified; those related to Gilbert's syndrome were in $16 \%$. Prevalence of polymorphic genotypes in icteric patients was $13.5 \%$ and in normal patients was $18.2 \%, p=0.08$. In $86.6 \%$ of white neonates - lowrisk genotypes, $13.4 \%$, Gilbert's genotypes, while 